\title{
Preparation and Bending Strength of Micro-Laminated TiC/Ti Composites
}

\author{
Tan Youde ${ }^{1,2}$, Cheng Xingwang ${ }^{1,2, *}, \mathrm{Xu} \mathrm{Ziqi}^{1,2}$ \\ ${ }^{1}$ School of Materials Science and Engineering, Beijing Institute of Technology, Beijing, China \\ ${ }^{2}$ National Key Laboratory of Science and Technology on Materials Under Shock and Impact, Beijing Institute of Technology, Beijing, China \\ Email address: \\ 2120161162@bit.edu.cn (Tan Youde), chengxw@bit.edu.cn (Cheng Xingwang), 1135698098@qq.com (Xu Ziqi) \\ ${ }^{*}$ Corresponding author
}

To cite this article:

Tan Youde, Cheng Xingwang, Xu Ziqi. Preparation and Bending Strength of Micro-Laminated TiC/Ti Composites. Science Discovery. Vol. 6, No. 4, 2018, pp. 257-262. doi: 10.11648/j.sd.20180604.15

Received: June 23, 2018; Accepted: August 4, 2018; Published: August 10, 2018

\begin{abstract}
Ceramic materials have some shortcomings such as intrinsic brittleness and poor reliability, which limit the application in the field of structure. The laminated composites are composed by ceramics and metals according to the principle of bionics, and the advantages of metals and ceramics can be excellently utilized to greatly improve the toughness and reliability of ceramic materials. At present, most of the methods are to prepare ceramic layers by using ceramic powders. This methods have the disadvantages of long preparation cycles and weak interface between ceramic and metal, and thus it is necessary to find a new synthesis method. Micro-laminated TiC/Ti composites were prepared by in-situ synthesis using Ti foils and graphite papers in this paper. The SPS and hot-pressing sintering were used respectively, and the microstructure and mechanical properties of the composite were compared. By comparison, the layered structure was obviously found after SPS and hot-press sintering at $1400^{\circ} \mathrm{C}$, but much residues $\mathrm{C}$ affected the mechanical properties; Due to the middle of Ti melting after SPS sintering at $1500^{\circ} \mathrm{C}$, the laminated structure could not be completely formed resulting in low strength. The laminated structure was well formed by hot-pressing sintering at $1500^{\circ} \mathrm{C}$, and the micro-laminated $\mathrm{TiC} / \mathrm{Ti}$ composite exhibited bending strength of $665.8 \pm 18 \mathrm{MPa}$ and fracture toughness of $23.81 \pm 1.2 \mathrm{MPa} \cdot \mathrm{m}^{1 / 2}$. However, the two sintering methods still need to be optimized.
\end{abstract}

Keywords: In-Situ Synthesis, Micro-Laminate Composites, Microstructure, Fracture Toughness

\section{TiC/Ti微叠层复合材料制备工艺和弯曲强度的研究}

\author{
谭友德 ${ }^{1,2}$, 程兴旺 ${ }^{1,2 *}$, 徐子祁 ${ }^{1,2}$ \\ ${ }^{1}$ 材料学院, 北京理工大学, 北京, 中国 \\ 2冲击环境材料技术重点实验室, 北京理工大学, 北京, 中国
}

邮箱:

2120161162@bit.edu.cn（谭友德）, chengxw@bit.edu.cn(程兴旺）, 1135698098@qq.com(徐子祁)

摘要: 陶瓷材料具有本征脆性和可靠性差等缺点, 制约陶瓷材料在结构领域的应用。通过仿生学原理将陶瓷与金属复
合成的叠层材料, 可以很好地利用金属和陶瓷的优点, 大提高陶瓷材料的韧性和可靠性。目前大多数方法是采用的
陶瓷粉体制备陶瓷层, 这种方法具有制备周期长并且陶瓷和金属的界面结合薄弱等缺点, 需要寻找新的合成方法。本
文以 $\mathrm{Ti}$ 䈃和石墨纸为原料, 采用原位合成法制备 $\mathrm{TiC} / \mathrm{Ti}$ 微叠层复合材料, 分别采用了 $\mathrm{SPS}$ 和热压烧结, 并且对比研究了
两者的微观组织和力学性能。通过对比发现, 在 $1400^{\circ} \mathrm{C}$, SPS和热压烧结后的样品有明显的层状结构, 但是有大量
的 $\mathrm{C}$ 残留, 并且影响力学性能; 在 $1500^{\circ} \mathrm{C下,} \mathrm{SPS}$ 烧结后出现 $\mathrm{Ti}$ 熔化, 不能形成完整的叠层结构, 导致强度低, 热压烧 
结后形成的叠层结构较好, 弯曲强度为 $(665.8 \pm 18) \mathrm{MPa}$, 断裂韧性(23.81 \pm 1.2$) \mathrm{MPa} \cdot \mathrm{m}^{1 / 2}$, 力学性能最佳。但是, 两种烧 结方法仍需要优化。

关键词: 原位合成, 微叠层复合材料, 微观组织, 断裂韧性

\section{1. 引言}

碳化钛 $(\mathrm{TiC})$ 陶瓷具有高硬度、高熔点、耐磨损、热 稳定性能好等独特的性能, 广泛应用于刀具涂层、钛合 金的增强相、核反应堆结构件等方面[1-2]。但是由于 $\mathrm{TiC}$ 陶瓷的本征脆性导致室温断裂韧性 $\left(\mathrm{K}_{\mathrm{IC}}\right)$ 低 $\left(\mathrm{K}_{\mathrm{IC}}\right.$ 约 $\left.5 \mathrm{MPa} \cdot \mathrm{m}^{1 / 2}\right)[3]$, 在很多领域的应用受到了限制。这是因 为 $\mathrm{TiC}$ 是一种陶瓷相, 陶瓷内部的化学键具有方向性, 导 致陶瓷受到外力时无法通过塑性变形吸收外加载荷能量, 只能依靠裂纹萌生和扩展而产生新的表面能来抵抗外加 载荷, 因此, 要提高陶瓷材料的韧性, 需要提高陶瓷裂 纹扩展的表面能和增加裂纹扩展路径。随着仿生学的发 展, 通过一些天然生物结构材料如贝壳珍珠层得到启发, 设计出了脆性层与韧性层相互交替排列的叠层复合材料, 通过叠层的多界面效应增加裂纹扩展的路径, 大大的提 高材料的㓞性。20世纪 90 年代, Clegg等[4]人制备出了碳 化硅 $(\mathrm{SiC})$ /石墨 $(\mathrm{C})$ 微叠层复合材料, 发现该材料断裂韧 性达到了 $15 \mathrm{MP} \cdot \mathrm{m}^{1 / 2}$, 约是普通的 $\mathrm{SiC}$ 陶瓷材料的 4 倍, 并 发表于《Nature》杂志。随后相继出现许多陶瓷增韧体系 的微叠层复合材料, 通常陶瓷层选择氧化铝 $\left(\mathrm{Al}_{2} \mathrm{O}_{3}\right)$ 陶瓷, 氮化铝 $(\mathrm{AlN})$ 陶瓷, $\mathrm{TiC}$ 陶瓷, $\mathrm{SiC}$ 陶瓷和硼化钑 $\left(\mathrm{B}_{4} \mathrm{C}\right)$ 陶 瓷等, 韧性层选择钛 $(\mathrm{Ti})$, 镍 $(\mathrm{Ni})$, 铝 $(\mathrm{Al})$, 石墨 $(\mathrm{C})$ 和六 方氮化嗍(h-BN)等。由于金属的韧性比石墨和h-BN要高, 因此选用金属与陶瓷复合形成的金属/陶瓷微叠层复合 材料的增韧效果更明显, 并且在防护领域具有很好的应 用前景[5]。

目前很多种类金属/陶瓷复合材料已经被制备出来。 2000年, 罗永明等[6]采用凝胶铸造的方法得到 $\mathrm{SiC}$ 基片后 进行喷涂金属铇(W)粉, 通过热压烧结制备出 $\mathrm{SiC} / \mathrm{W}$ 叠层 复合材料, 测得断裂㓞性达 $8.1 \mathrm{MPa} \cdot \mathrm{m}^{1 / 2}$, 是 $\mathrm{SiC}$ 陶瓷块体 的2倍。金海云等[7]-[8]人采用流延法成型和(放电等离子 烧结)SPS的制备方法成功制备出 AlN/Ti、AlN/Ni等陶瓷/ 金属叠层复合材料, 其断裂韧性分别达到了 $8.54 \mathrm{MPa} \cdot \mathrm{m}^{1 / 2}$ 和 $9.32 \mathrm{MPa} \cdot \mathrm{m}^{1 / 2}$ 。Huang等 [9]人选择了铝粉和 $\mathrm{Al}_{2} \mathrm{O}_{3}$ 陶瓷片, 在较低温度下采用热压烧结, 制备出 $\mathrm{Al}_{2} \mathrm{O}_{3} / \mathrm{Al}$ 叠层复合材 料, 得到弯曲强度可达 $387 \mathrm{MPa}$ 、断裂韧性 $10.5 \mathrm{MPa} \cdot \mathrm{m}^{1 / 2}$ 。 Song等 [10]利用 $\mathrm{Al}_{2} \mathrm{O}_{3}$ 陶瓷板, 通过改变陶瓷表面形状, 与 金属钼(Mo)进行热压烧结制备出 $\mathrm{Al}_{2} \mathrm{O}_{3} / \mathrm{Mo}$ 叠层复合材料, 得出锯齿状界面的自锁功能有利于提高界面结合强度, 并 且材料的㓞性, 断裂功和弯曲强度分别可达到 $8.0 \mathrm{MPa} \cdot \mathrm{m}^{1 / 2}$ 、 $1179 \mathrm{~J} \cdot \mathrm{m}^{-2}$ 和 $293 \mathrm{MPa}$, 分别是单片 $\mathrm{Al}_{2} \mathrm{O}_{3}$ 陶瓷的 2.1 倍, 6.6 倍和 1.2 倍。 $\mathrm{Wu}$ 等 $[11]$ 人利用 $\mathrm{T} i$ 粉和 $\mathrm{B}_{4} \mathrm{C}$ 粉流延成型后进行 热压烧结, 制备出 $\mathrm{B}_{4} \mathrm{C} / \mathrm{Ti}$ 金属/陶瓷微叠层复合材料, 其断 裂韧性达到 $9.2 \mathrm{MPa} \cdot \mathrm{m}^{1 / 2}$ 。Launey等[12]人采用冷冻铸造法 制备出氧化铝陶瓷骨架, 利用压力浸渍的方法, 将铝-硅 (Al-Si)合金熔渗到陶瓷骨架中, 制备出 $\mathrm{Al}_{2} \mathrm{O}_{3} / \mathrm{Al}-\mathrm{Si}$ 合金叠
层复合材料, 并测得其拉伸强度 $300 \mathrm{MPa}$, 断裂㓞性高达 $40 \mathrm{MPa} \cdot \mathrm{m}^{1 / 2}$, 其力学性能和铝合金相近。Claudio Ferraro 等[13]对这种冷冻铸造后渗透合金的方法进行了系统的研 究和分析。沈平等 [14]人采用和冷冻铸造的方法制备出 $\mathrm{TiC} / \mathrm{Al}$ 叠层复合材料, 其弯曲强度达 $355 \pm 3 \mathrm{MPa}$ 和最高断 裂韧性达 $81 \pm 2 \mathrm{MPam}^{1 / 2}$, 并发现随着TiC陶瓷含量的增加, 断裂㓞性呈现逐渐减弱的趋势。大多数制备陶瓷层的方法 采用的原料是陶瓷粉体, 然后经过成型、烧结得到微叠层 复合材料。虽然采用粉末烧结的方法有制备工艺成熟, 设 备要求低等优点, 但是也存在一些不足:1).制备过程陶瓷 粉体会难免产生团聚; 2). 单层厚度很难保持一致和均匀, 影响力学性能; 3). 陶瓷成型到烧结期间周期较长, 成型工 艺过程复杂; 4).陶瓷表面具有惰性, 与金属结合的界面强 度相对较低。因此需要寻找新的制备陶瓷/金属微叠层复 合材料。原位合成法主要是在金属基体上通过化学反应形 成新的陶瓷相, 从而改善金属的性能。这种方法在金属基 复合材料应用较为广泛, 界面处结合紧密, 界面结合强度 好[15]。然而这种方法应用于制备金属/陶瓷微叠层复合材 料鲜有报道。

针对目前金属/陶瓷叠层复合材料中陶瓷层的制备存 在的问题, 本文采用箔片为原料, 采用原位反应方法制备 出陶瓷层, 而后与残留的金属形成金属/陶瓷叠层复合材 料。该方法不需要提前制备陶瓷和金属粉末, 用 $\mathrm{T}$ 簿片和 石墨纸为原料, 利用 $\mathrm{Ti}$ 与 $\mathrm{C}$ 原子之间扩散反应得到 $\mathrm{TiC}$ 陶瓷 层, 然后 $\mathrm{TiC}$ 层与残留的金属 $\mathrm{Ti}$ 的形成金属/陶瓷微叠层复 合材料。本文采用SPS和热压烧结的方法, 对比研究两种 烧结工艺的得到的组织和力学性能, 探索制备 $\mathrm{TiC} / \mathrm{Ti}$ 微叠 层复合材料较佳的工艺参数。

\section{2. 材料和方法}

本次研究采用的原材料是工业 $\mathrm{Ti}$ 箔(纯度 $99.5 \%$, 厚 $0.1 \mathrm{~mm}$ )和石墨纸(纯度 $99.5 \%$, 厚 $0.02 \mathrm{~mm}$ ), Ti簿和石墨纸 切成直径 $50 \mathrm{~mm}$ 的小圆片, 用 600 号的砂纸对 Ti簿进行打磨, 然后用 $5 \%$ 的氢氟酸(HF)溶液浸泡 5 $10 \mathrm{~min}$ 后用去离子水 和乙醇超声清洗2 3次, 最后进行干燥。取 $\mathrm{Ti}$ 簿和石墨纸 各100片, 交替叠加放入石墨模具中。采用SPS烧结炉 (Sojitz Machinery)进行真空烧结, 温度为 $1400^{\circ} \mathrm{C}$ 和 $1500^{\circ} \mathrm{C}$, 升温速率 $150^{\circ} \mathrm{C} / \mathrm{min}$, 保温时间 $15 \mathrm{~min}$, 压力 $20 \mathrm{MPa}$, 随炉 冷却, 取出样品。采用R-C-ZKQY-07型热压烧结炉进行真 空热压烧结, 烧结温度为 $1400^{\circ} \mathrm{C}$ 和 $1500^{\circ} \mathrm{C}$, 加热速率 $10^{\circ} \mathrm{C} / \mathrm{min}$, 烧结压力为 $20 \mathrm{MPa}$, 烧结时间为 $2 \mathrm{~h}$, 随炉冷却, 取出样品。

用 X射线衍射仪(XRD)对试样横截面进行分析晶体 结构和相的成分, 使用型号为Bruker D8Advance(德国, 布鲁克), 测试使用的靶材为铜靶 ( $\mathrm{K} \alpha, \mathrm{X}$ 射线波长 $\lambda=0.15418 \mathrm{~nm})$, 电压 $40 \mathrm{kV}$, 电流 $35 \mathrm{~mA}$, 连续扫描, 扫描 
速度为 $5^{\circ} / \mathrm{min}$, 扫描测试范围 $10^{\circ} \sim 90^{\circ}$, 扫描步长为 $0.02 \%$ step。采用背散射扫描电镜(SEM)和能谱(EDS)对试 样的微观结构和元素分布进行表征。采用万能试验机 (INSTRO) 在室温下进行三点弯曲强度(TPB)和断裂韧性 的测试, 万能试验机型号为WDW-200D。测试三点弯曲 强度的试样尺寸是 $3 \mathrm{~mm} \times 4 \mathrm{~mm} \times 20 \mathrm{~mm}$, 其中叠层厚度是 $3 \mathrm{~mm}$, 加载速度是 $0.5 \mathrm{~mm} / \mathrm{min}$, 测试五个样, 取平均值; 测试断裂韧性采用单边开口刀法, 试样尺寸是 $4 \mathrm{~mm} \times 2 \mathrm{~mm} \times 20 \mathrm{~mm}$, 其中叠层厚度是 $4 \mathrm{~mm}$, 切口垂直于 叠层方向, 切口深度 $2 \mathrm{~mm}$, 加载速度是 $0.05 \mathrm{~mm} / \mathrm{min}$, 跨 距是 $16 \mathrm{~mm}$, 测试五个样, 取平均值, 根据下列公式(1) 出断裂韧性 $\mathrm{K}_{\mathrm{IC}}$ 的数值:

$$
\mathrm{K}_{\mathrm{IC}}=\frac{3 \mathrm{~F}_{\max } \mathrm{L}}{2 \mathrm{BW}^{2}} \sqrt{\mathrm{a}} \times \mathrm{f}\left(\frac{\mathrm{a}}{\mathrm{W}}\right)
$$

其中, $\mathrm{F}_{\max }$ 表示最大载荷, L表示试样跨距, B表示试 样厚度, $\mathrm{W}$ 表示试样宽度, $\mathrm{a}$ 表示试样中间切口深度, 函 数 $\mathrm{f}(\mathrm{a} / \mathrm{W})$ 的计算过程如公式(2)[16]下:

$f\left(\frac{a}{W}\right)=1.96-2.75\left(\frac{a}{w}\right)+13.66\left(\frac{a}{w}\right)^{2}-23.98\left(\frac{a}{w}\right)^{3}+25.22\left(\frac{a}{w}\right)^{4}$

在本实验中, $\mathrm{L}, \mathrm{B}, \mathrm{W}, \mathrm{a}$ 分别对应的是 $16 \mathrm{~mm}, 4 \mathrm{~mm}$, $3 \mathrm{~mm}, 2 \mathrm{~mm}$ 。

\section{3. 结果与讨论}

\section{1. 微观形貌和结构组成}

下图1是采用 SPS 和热压烧结后得到的 $\mathrm{TiC} / \mathrm{Ti}$ 复合材 料的XRD衍射图谱。根据衍射峰和标准PDF卡片的比对得 出, 在 $1400^{\circ} \mathrm{C}$ 下烧结后, 该复合材料由 $\mathrm{TiC} 、 \alpha-\mathrm{Ti}$ 和 $\mathrm{C}$ 三种 相组成, 结合扫描电镜图片图2(a)和图2(c) 可以看出, 层状

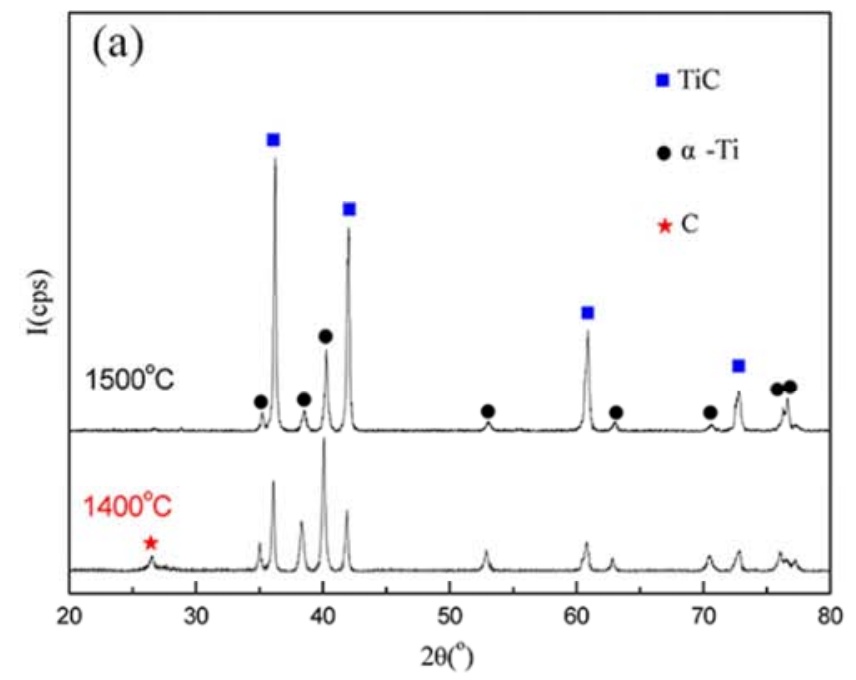

结构比较明显, 结合能谱分析得出, 亮且偏白的部分是未 反应完全的 $\alpha-\mathrm{Ti}$, 厚度约 $50 \mu \mathrm{m}$, 灰色较暗部分是原位形成 的 $\mathrm{TiC}$ 反应层, 厚度约为 $15 \mu \mathrm{m}$, 黑色部分是残留的石墨, 厚约 $10 \mu \mathrm{m}$, 在 $1400^{\circ} \mathrm{C}$ 烧结后发现, SPS烧结和热压烧结得 到的层状结构相似, 都残留大量的石墨; 通过图1展示在 $1500^{\circ} \mathrm{C}$ 下烧结后, $\mathrm{C}$ 的衍射峰基本消失, 只有 $\mathrm{TiC}$ 与 $\alpha-\mathrm{Ti}$ 的衍射峰, 从扫描电镜图2(b)发现, $1500^{\circ} \mathrm{C}$ 下SPS烧结后, $\mathrm{TiC}$ 层部分相互挤压在一起, 层状结构不明显, 出现边缘 有层状, 中心无层状结构, 还有很少的石墨残留, 产生的 原因是中心部分 Ti出现融化, 得不到层状结构; 通过图2(d) 发现, $1500^{\circ} \mathrm{C}$ 下热压烧结得到的层状结构明显, $\mathrm{Ti}$ 层厚度 大约 $30 \mu \mathrm{m}, \mathrm{TiC}$ 层厚度约 $50 \mu \mathrm{m}, \mathrm{TiC}$ 层中会发现部分 $\mathrm{Ti}$ 渗 透。

结合XRD图和 SEM分析得出, 在 $1400^{\circ} \mathrm{C}$ 和 $1500^{\circ} \mathrm{C}$ 温度下, $\mathrm{Ti}$ 与 $\mathrm{C}$ 发生了扩散反应, 形成了 $\mathrm{TiC}$ 陶瓷。根据 $\mathrm{XRD}$ 衍射峰对比发现, $1500^{\circ} \mathrm{C}$ 下 $\mathrm{TiC}$ 的衍射峰变宽, $\mathrm{Ti}$ 的衍射峰强度变低, 并且没有 $\mathrm{C}$ 的衍射峰, 说明 $1500^{\circ} \mathrm{C}$ 更有利于 TiC形成。另外出现熔化的现象可能是 SPS 烧结 时是材料结合界面的放电效应和 Ti的导热性不佳, 内外 温度不均导致的。采用的热压烧结后的 $\mathrm{TiC} / \mathrm{Ti}$ 微叠层复 合材料的XRD检测结果很相似, 没有其他相生成。通过 SEM结果(图2(c)和图2(d))发现, 采用热压在 $1400^{\circ} \mathrm{C}$ 的条 件下, 可以看见明显的层状结构, 但仍然有部分 $\mathrm{C}$ 残留; 在 $1500^{\circ} \mathrm{C}$ 下, 几乎没有 $\mathrm{C}$ 的残余, 层状结构非常明显, 界面没有发现裂缝和孔洞, 也没有出现熔化的现象。此 外, 部分 $\mathrm{Ti}$ 渗入到了 $\mathrm{TiC}$ 陶瓷层的原因可能是在冷却时, $\mathrm{TiC}$ 中析出了 $\mathrm{Ti}$, 或者是 $\mathrm{TiC}$ 存在缝隙, $\mathrm{Ti}$ 被挤压到了 $\mathrm{TiC}$ 基体中。

通过SPS和热压烧结的结果对比得知, SPS烧结温 度低会导致 C有残留, 烧结温度高导致 Ti出现熔化。采 用热压烧结时, 温度低也同样会导致 $\mathrm{C}$ 残留, 温度高得 到的叠层结构比较明显, 但是会使 $\mathrm{Ti}$ 熔渗到 $\mathrm{TiC}$ 陶瓷基 体中。

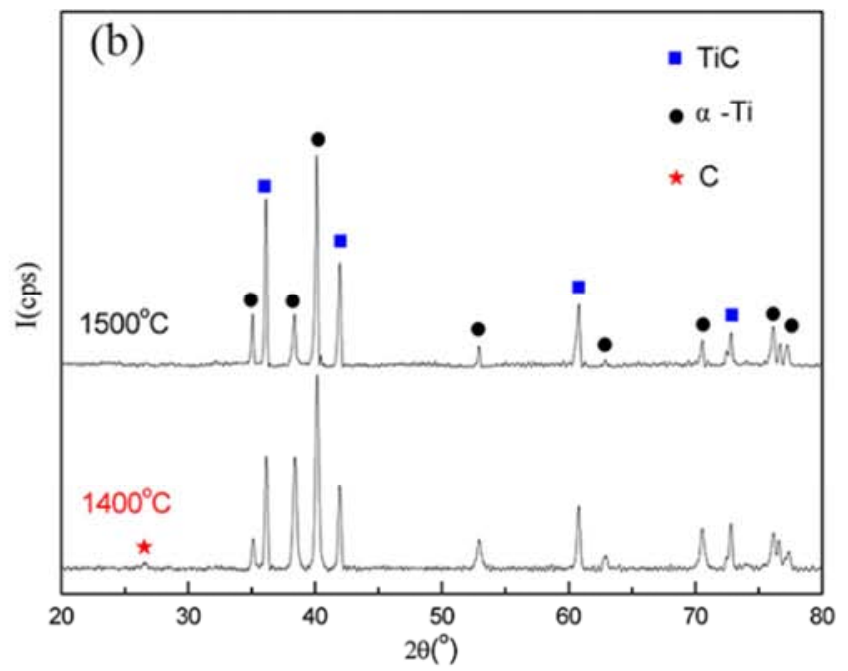

图1 烧结后的XRD衍射图谱: (a)SPS烧结, (b)热压烧结。 


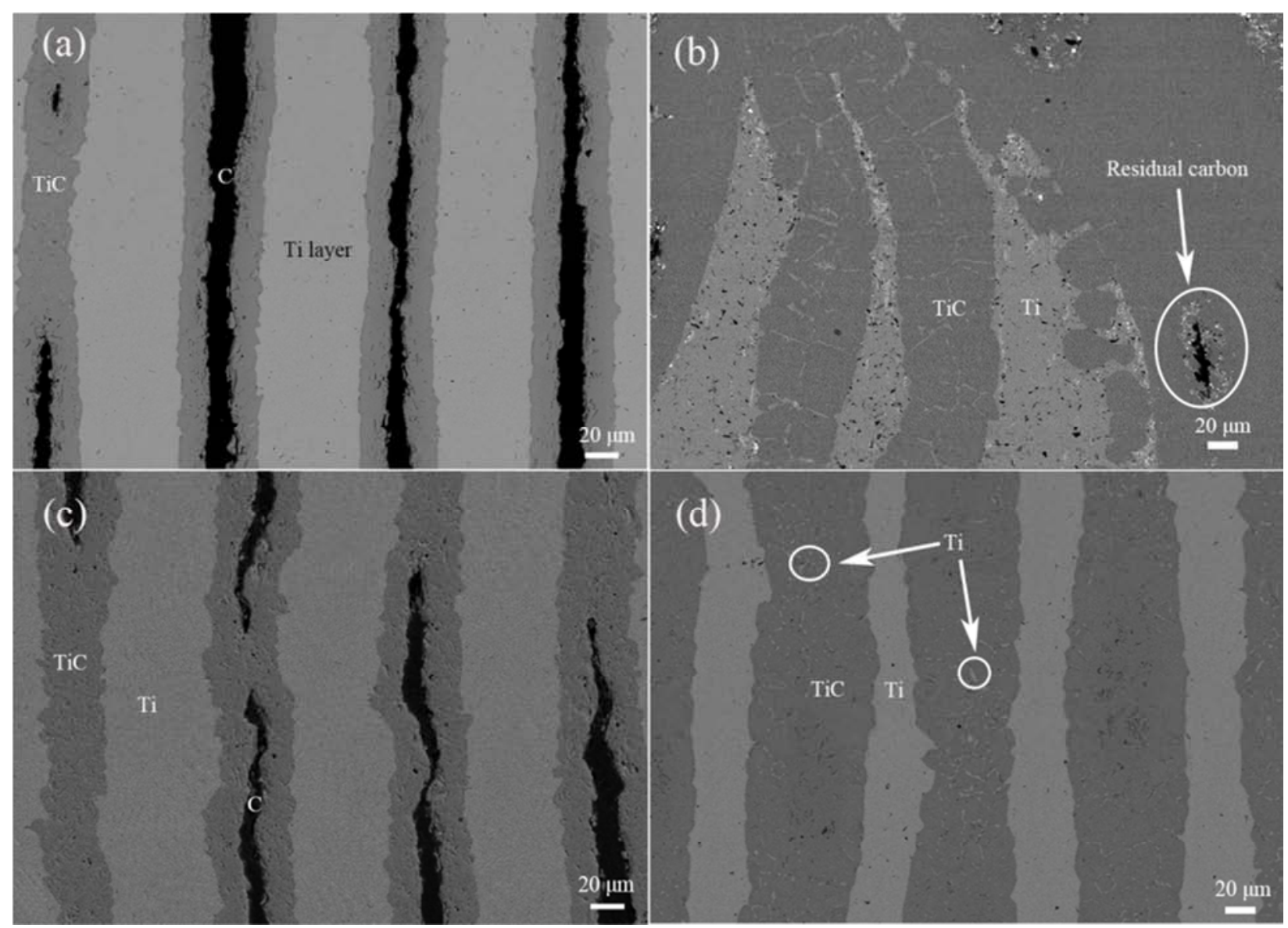

图2 扫描电镜图:(a) $1400^{\circ} \mathrm{C}$ 下SPS烧结; (b) $1500^{\circ} \mathrm{C}$ 下SPS烧结; (c) $1400^{\circ} \mathrm{C}$ 热压烧结; (d) $1500^{\circ} \mathrm{C}$ 热压烧结。

\section{2. 弯曲强度和断裂韧性}

本实验采用三点弯曲法对复合材料的弯曲强度和断 裂韧性进行测试。通过测试发现, 采用SPS 和热压在 $1400^{\circ} \mathrm{C}$ 烧结后样品层间分裂严重, 无法加工测试样件, 因 此无法进行力学性能测试。这是由于残留的石墨纸的力学 性能差, 复合材料中的 $\mathrm{TiC}$ 层结合非常薄弱, 从而影响复 合材料整体的力学性能。当温度在 $1500^{\circ} \mathrm{C}$ 下, 采用SPS烧 结的复合材料的抗弯强度 $(84.1 \pm 22) \mathrm{MPa}$, 无法加工断裂韧 性的样件; 采用热压法烧结得到复合材料的弯曲强度为 $(665.8 \pm 18) \mathrm{MPa}$, 断裂韧性 $(23.81 \pm 1.2) \mathrm{MPa} \cdot \mathrm{m}^{1 / 2}$, 弯曲强度 与无压烧结 $\mathrm{TiC}$ 陶瓷大致相等, 断裂韧性比一般的 $\mathrm{TiC}$ 陶瓷 高2-4倍[17]。说明在一定温度和压力下, 热压法制备叠层 复合材料在结构和性能优于SPS烧结法。

图3a显示的 $1500^{\circ} \mathrm{C}$ 下热压烧结后, 通过三点弯曲断裂 后的裂纹扩展图。由图发现, 该图有主裂纹和一些微小裂 纹组成。其中主裂纹呈现锯齿状, 裂纹在 $\mathrm{TiC}$ 层和 $\mathrm{Ti}$ 层之 间出现了偏转; 在裂纹尖端区域发现, 裂纹直接穿过 $\mathrm{Ti}$ 层且Ti层未完全断裂, 说明裂纹出现了桥联; 在部分碳化 钛层中出现分层的现象, 未发生在界面处, 说明 $\mathrm{TiC}$ 与 $\mathrm{Ti}$ 的界面结合比较强。裂纹偏转、桥联和分层均有利于提高 复合材料的整体的断裂㓞性。此外, 分层区域未发生在 TiC
与 $\mathrm{Ti}$ 的界面, 说明 $\mathrm{TiC}$ 与 $\mathrm{Ti}$ 具有较高的界面结合强度。图 $3 \mathrm{~b}$ 表示的是 $1500^{\circ} \mathrm{C}$ 下热压烧结后断口形貌, 由图可以发现 $\mathrm{TiC}$ 层断裂面平整, 是典型的脆性断裂, $\mathrm{Ti}$ 层出现山脊状 和韧窝, 是典型的㓞性断裂。此图还可以发现 TiC层中间 部分有平行叠层方向的裂纹, 界面处未发现脱落的情况, 进一步证明 TiC 与 Ti的界面结合力强, 说明了这种原位反 应法制备的叠层 $\mathrm{TiC} / \mathrm{Ti}$ 复合材料的界面具有很强的结合力。

图3c 和图 $3 \mathrm{~d}$ 分别表示 $1500^{\circ} \mathrm{C}$ 下热压烧结后的弯曲强 度的应力-应变曲线和断裂韧性的载荷-位移曲线, 通过图 $3 \mathrm{c}$ 和图 $3 \mathrm{~d}$ 可以看出, 该复合材料的应力-应变曲线和载荷位移曲线与纯块体陶瓷材料不同 [18], 没有出现载荷急剧 下降的情况, 呈现非脆性断裂, 说明该复合材料有一定的 可靠性和金属层有一定的承载能力。

结合微观组织和力学性能分析, 对SPS烧结和热压烧 结两种烧结方法进行了比较后发现: SPS烧结具有升温速 率快, 烧结时间短, 效率高等优势, 但是由于烧结时间短, 很难通过扩散反应形成较好的叠层结构; 热压烧结可以通 过控制一定工艺参数得到较佳的微叠层复合材料, 但是也 存在升温速率较慢, 烧结时间长的问题, 另外烧结温度较 低导致 C残留, 烧结温度高导致Ti渗透等问题需要解决。 

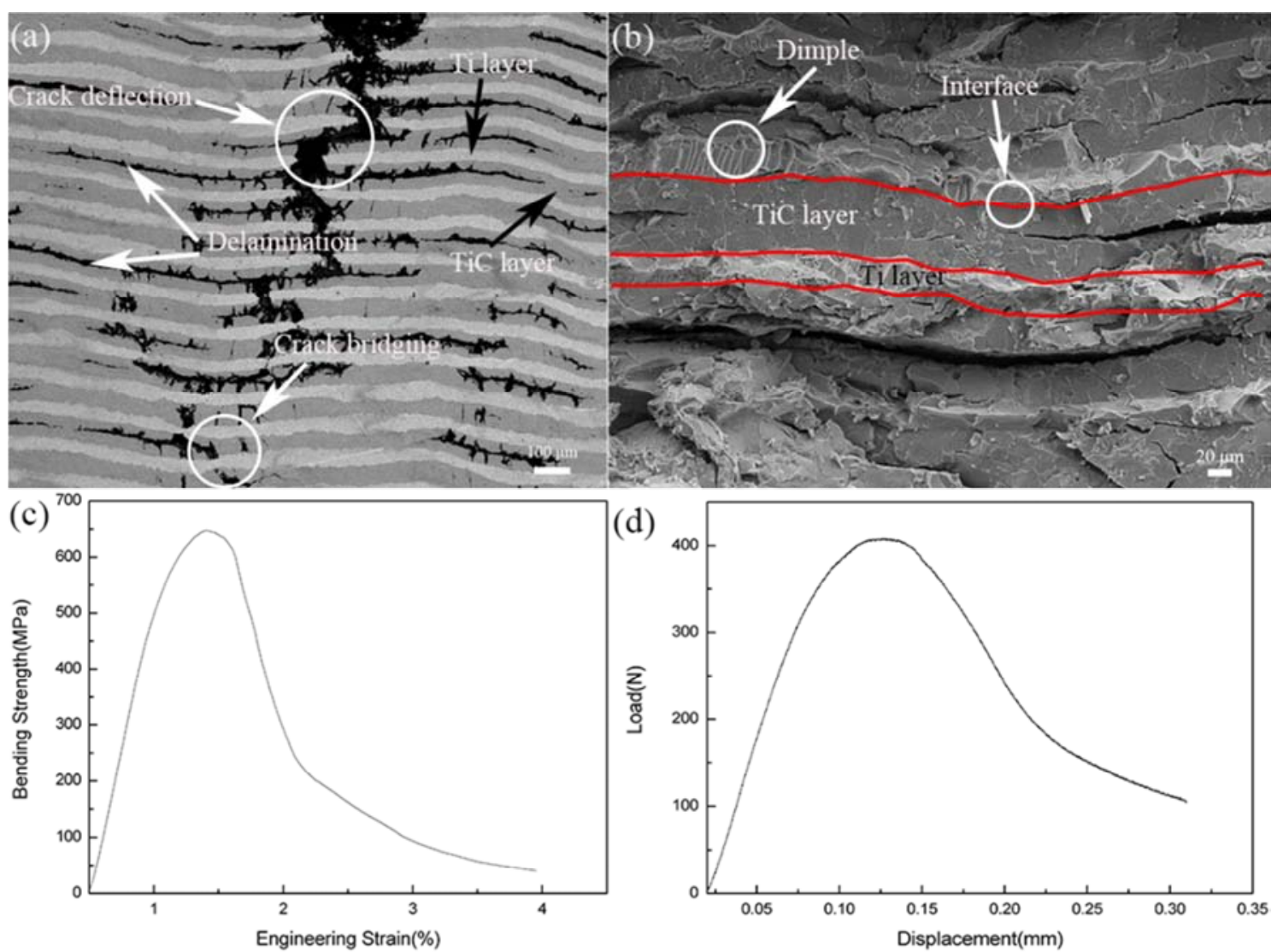

图3 (a)裂纹扩展图; (b)断口形貌图; (c)弯曲应力-应变曲线; (d)载荷-位移曲线。

\section{4. 结论}

采用 $\mathrm{Ti}$ 箔和石墨纸为原料, 通过加热加压的方式, 原 位合成方法制备 $\mathrm{TiC} / \mathrm{Ti}$ 微叠层复合材料。通过比较了相同温 度下SPS和热压烧结后的产物, 发现在 $1400^{\circ} \mathrm{C}$ 下烧结的复 合材料中具有很多 $\mathrm{C}$ 残留, 影响力学性能; 在 $1500^{\circ} \mathrm{C}$ 下, 采用热压烧结方法得到的微叠层复合材料的微观组织和力 学性能更好, 弯曲强度为 $(665.8 \pm 18) \mathrm{MPa}$, 断裂韧性 $(23.81 \pm 1.2) \mathrm{MPa} \cdot \mathrm{m}^{1 / 2}$ 。弯曲强度与 $\mathrm{TiC}$ 大致相等, 断裂韧性 却大大的提高。另外SPS烧结方法需要继续探索更佳的工艺。 $\mathrm{SPS}$ 烧结需要解决的问题是如何让 C无残留, 同时 $\mathrm{Ti}$ 不能熔 化; 热压烧结是解决的问题是Ti的渗透和生产效率低下。 综上所述, SPS和热压烧结方法仍然不断地改进和优化。

\section{致谢}

本文为国家自然科学基金(基金号:51271036)的阶段 性成果之一。

\section{参考文献}

[1] Yu H L, Zhang W, Wang H M, et al. In-situ synthesis of $\mathrm{TiC} / \mathrm{Ti}$ composite coating by high frequency induction cladding $[\mathrm{J}]$. Journal of Alloys and Compounds, 2017, 701:244-255.
[2] Chen D, Liu D, Liu Y, et al. Microstructure and fretting wear resistance of $\gamma / \mathrm{TiC}$ composite coating in situ fabricated by plasma transferred arc cladding $[J]$. Surface \& Coatings Technology, 2014, 239(239):28-33.

[3] Deng J, Can T, Sun J. Microstructure and mechanical properties of hot-pressed $\mathrm{Al}_{2} \mathrm{O}_{3} / \mathrm{TiC}$ ceramic composites with the additions of solid lubricants[J]. Ceramics International, 2005, 31(2):249-256.

[4] Clegg W J, Kendall K, Alford N M, et al. A simple way to make tough ceramics[J]. Nature, 1990, 347(6292):455-457.

[5] Adharapurapu R R, Vecchio K S, Jiang F, et al. Fracture of $\mathrm{Ti}-\mathrm{Al}_{3} \mathrm{Ti}$ metal-intermetallic laminate composites: Effects of lamination on resistance-curve behavior[J]. Metallurgical \& Materials Transactions A, 2005, 36(11):3217-3236.

[6] 罗永明,袁广江. SiC/W层状复合材料的制备工艺与力学性 能[J].炭素技术,2000(4):43-46。

[7] Zhang T, Li W Z, Jin H Y, et al. The Mechanical Properties and Fracture Characteristics of AlN/Ti Laminated Composites[J]. Materials Science Forum, 2010, 658: 348-351.

[8] Jin $\mathrm{H} \mathrm{Y,} \mathrm{Jin} \mathrm{P,} \mathrm{Li} \mathrm{Y} \mathrm{F,} \mathrm{et} \mathrm{al.} \mathrm{Fabrication} \mathrm{and} \mathrm{Fracture}$ Characteristics of AlN/Ni Laminated Composites[J]. Materials Science Forum, 2014, 804:183-186.

[9] Kangming Huang, Li W, Xie B, et al. Preparation and mechanical properties of $\mathrm{Al}_{2} \mathrm{O}_{3} / \mathrm{Al}$ laminated ceramic matrix composites[J]. Journal of Wuhan University of Technology, 2011, 26(5):891-896. 
[10] Song J, Zhang Y, Fan H, et al. Design of interfaces for optimal mechanical properties in $\mathrm{Al}_{2} \mathrm{O}_{3} / \mathrm{Mo}$ laminated composites[J]. Journal of the European Ceramic Society, 2015, 35(3):1123-1127.

[11] Wu, Chao; LI, Yunkai; XIE, Shihui. Micro-structure, mechanical properties and comparison of monolithic and laminated $\mathrm{Ti}-\mathrm{B}_{4} \mathrm{C}$ composite with $\mathrm{Al}$ doped[J].Journal of Alloys and Compounds, 2018, 733: 1-7.

[12] Launey M E, Munch E, Alsem D H, et al. A novel biomimetic approach to the design of high-performance ceramic-metal composites[J]. Journal of the Royal Society Interface, 2009, 7(46):741-753.

[13] Ferraro C, Meille S, Réthoré J, et al. Strong and tough $\mathrm{metal} /$ ceramic micro-laminates[J]. Acta Materialia, 2017, 144.
[14] Wang, Ya, et al. Developing high toughness and strength $\mathrm{Al} / \mathrm{TiC}$ composites using ice-templating and pressure infiltration[J]. Ceramics International, 2017, 43(4): 3831-3838.

[15] 吕维洁,郭相龙,王立强,等.原位自生非连续增强钛基复合材 料的研究进展 [J].航空材料学报,2014,34(4):139-146。

[16] Zhang Y, Cheng X, Cai H, et al. The effects of thickness of original $\mathrm{Ti}$ foils on the microstructures and mechanical properties of $\mathrm{Ti}_{2} \mathrm{Ni} / \mathrm{TiNi}$ laminated composites[J]. Materials Science \& Engineering A, 2017, 684:292-302.

[17] 刘阳,曾令可编.碳化钛陶瓷及应用[M].北京:化学工业出版 社,2008:14-15。

[18] 黄勇,汪长安.高性能多相复合陶瓷[M].北京:清华大学出版 社,2008:362-363。 